\title{
Luminescent Properties of Arylpolyene Organic Dyes and Cross-Conjugated Ketones Promising for Quantum Optics and Nanophotonics Applications
}

\author{
N. L. Naumova ${ }^{a}$ and I. A. Vasilyeva \\ Moscow State Pedagogical University, 119991, 29, Malaya Pirogovskaya, Moscow, Russia
}

\begin{abstract}
The spectral-luminescent properties of some dyes of substituted arylpolyenes and cross-conjugated ketones class in Shpolsky matrices, promising for using in solving quantum optics and nanophotonics, were studied.
\end{abstract}

Keywords: polyenes, Shpolsky matrix, single photons source, superfine resolution fluorescence microscopy

Dyes of polyenes class (compounds with an extended system of alternating single and double C-C bonds) are common and find a wide range of practical applications. The presence of linear conjugation bonds in polyenes molecules causes intense absorption of light energy in the near-UV and visible spectral range and a high luminescence quantum yield. In addition the synthesis of compounds with different numbers of $\mathrm{C}-\mathrm{C}$ bonds allows changing the spectral range of molecules. It should be noted that the ability of polyenes molecules for $\mathrm{J}$ - and H-aggregation allows to create molecular complexes (pairs and small ensembles) with specified intermolecular interaction properties [1]. All of the above indicates significant prospects of the polyenes class compounds in solving problems of quantum optics and nanophotonics, particularly in the following areas:

- Development of nonclassical light sources.

- Creation of optical storage materials.

- Development of doped media with a controllable photorefraction.

- Creation of molecular media for efficient conversion (transport) of electromagnetic radiation and electron excitation energy in condensed matter.

The prospects of new dyes for fluorescent microscopy of superfine spatial resolution, where the problem of finding new markers with controllable photophysical properties is one of the most pressing, should be noted separately [2].

In this paper we study the spectral properties of a wide range of substituted arylpolyenes and cross-conjugated ketones compounds [3-6]. Luminescence spectra and fluorescence excitation spectra, fluorescence quantum yield and excited state lifetimes were measured. The studies were carried out at cryogenic temperatures in Shpolsky matrices (n-octane), where the observation of fine spectra (zerophonon lines) is possible, allowing for quantitative analysis of intra- and intermolecular interactions [7].

\footnotetext{
${ }^{a}$ Corresponding author: n_1_naumova@mail.ru
} 
Small deviations from mirror symmetry of conjugate substituted arylpolyens spectra are explained by the joint manifestation of Herzberg-Teller (GT) and the Franck-Condon (FC) interactions. A quantitative estimation of FK / GT- parameters for certain compounds was made.

The measured spectra of cross-conjugated ketones compounds, showing significant differences from the mirror symmetry rule, were analyzed in the two-well adiabatic potential model developed by Osad'ko in [8]. In this model, the conjugate spectra can be calculated trough the numerical solution of the stationary Schrödinger equation with an adiabatic potential in the following form: $V(x)=-\Theta(\cos x-\eta \cos 2 x+\xi \sin x)$. The intensity of spectral lines corresponding to the individual electron-vibrational transitions were estimated by calculating the square of the overlap integral of the corresponding wave functions (see. Figure 1).
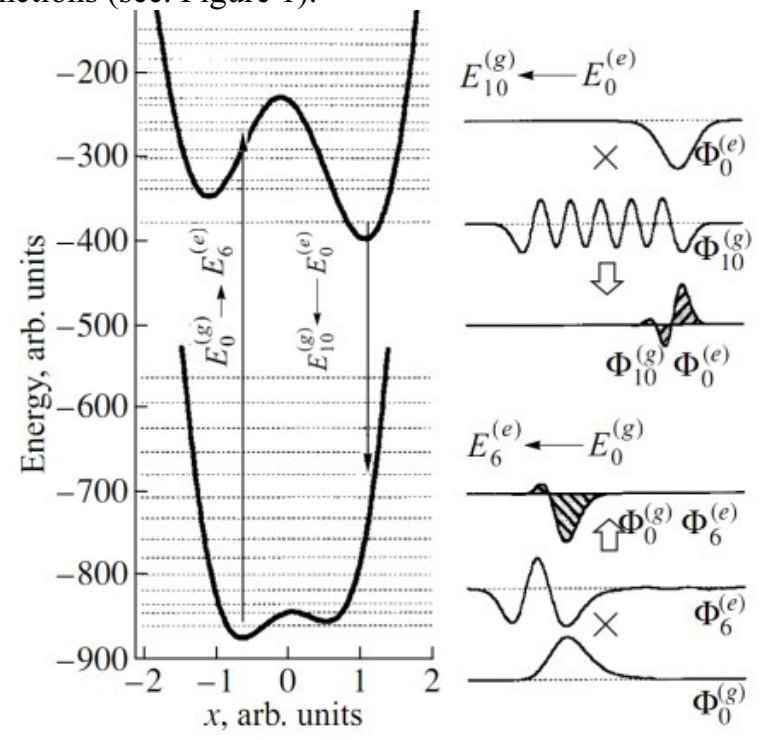

Figure 1. The two-well adiabatic potentials (left) of the ground and excited electronic states, allowing to describe the conjugated significally non-mirror-like spectra. The horizontal dotted lines represent the vibrational energy levels. Overlappings of the wave functions (right) define the transitions amplitudes (modified from [3]).

It is shown that this model can explain the significant anomalies in conjugate spectra (significant departure from mirror symmetry and a large gap in the frequency scale between the spectra).

The authors thank Prof. I.S. Osad'ko and Prof. A.V. Naumov for fruitful discussions. This work was supported by the Russian Foundation for Basic Research (grant 15-32-21100-mol-a-ved).

\section{References}

1. F. Wurthner, D. Debaerdemaeker, R. Wortmann, JACS 124, 9431 (2002)

2. A.V.Naumov, I.Yu.Eremchev, A.A.Gorshelev, Eur. Phys. J. D. 68, 348 (2014)

3. A.V. Naumov, N.L. Naumova, I.S. Osad'ko, Opt. Spectr. 91, 750 (2001)

4. N.L. Naumova et al., Opt. Spectr. 92, 426 (2002)

5. N.L. Naumova et al., Opt. Spectr. 98, 535 (2005)

6. N.L. Naumova et al., J. Lumin. 111, 37 (2005)

7. A.V. Naumov, Physics Uspekhi. 56. P.605 (2014)

8. I. S. Osad'ko, S. A. Kulagin, Opt. Spektr. 49, 290 (1980) 